\title{
Double-cell superstructure and vacancy ordering in tensile-strained metallic thin films of $\mathrm{Pr}_{0.50} \mathrm{Ca}_{0.50} \mathrm{CoO}_{3}$ on $\mathrm{LaAlO}_{3}$
}

\author{
Jessica Padilla-Pantoja, ${ }^{1}$ Xavier Torrelles, ${ }^{1}$ Jaume Gazquez, ${ }^{1}$ Juan Rubio-Zuazo, ${ }^{2,3}$ Javier Blasco, ${ }^{4}$ Javier Herrero-Martín, ${ }^{5}$ \\ and Jose Luis García-Muñoz $\oplus^{1, *}$ \\ ${ }^{1}$ Institut de Ciència de Materials de Barcelona, ICMAB-CSIC, Campus universitari de Bellaterra, E-08193 Bellaterra, Spain \\ ${ }^{2}$ SpLine Spanish CRG Beamline at the ESRF Grenoble cedex ESRF, BP 220-38043, France \\ ${ }^{3}$ Instituto de Ciencia de Materiales de Madrid, ICMM-CSIC, 28049 Madrid, Spain \\ ${ }^{4}$ Instituto de Ciencia de Materiales de Aragón, CSIC-Univ. de Zaragoza, 50009 Zaragoza, Spain \\ ${ }^{5}$ ALBA Synchrotron Light Source, 08290 Cerdanyola del Vallès, Barcelona, Spain
}

(Received 12 March 2019; revised manuscript received 22 July 2019; published 10 October 2019)

\begin{abstract}
The Pr-based cobaltate $\mathrm{Pr}_{0.5} \mathrm{Ca}_{0.5} \mathrm{CoO}_{3}$ (PCCO) presents in bulk form a singular simultaneous valence and spin-state transition that turns the metallic state into insulator, and displays a large and ultrafast photoresponse in the insulating phase. Epitaxial thin films of PCCO have been grown by deposition on $\mathrm{LaAlO}_{3}(001)$ (LAO) substrate, chosen to minimize the mismatch with the film. The films grow epitaxially with two times the substrate periodicity $\left(2 a_{0} \times 2 a_{0} \times 2 a_{0}\right)$ and the long perovskite axis perpendicular to the surface. We report a reduction of the structural symmetry from Pnma (bulk) to $P 2_{1} 2_{1} 2_{1}$ (film). The $P 2_{1} 2_{1} 2_{1}$ symmetry revealed by synchrotron $\mathrm{x}$ ray remains at low temperatures. These PCCO films are metallic, and ferromagnetic below $T_{\mathrm{C}}=170 \mathrm{~K}$, confirming the stabilization of excited $\mathrm{Co}^{3+}$ spin states and the suppression of the concurrent Co spin-state, valence, and metal-insulator transitions. $Z$-contrast imaging and electron-energy-loss spectroscopy elemental maps reveal long-range ordered oxygen vacancy planes unexpectedly stacking parallel to the interface, in spite of the tensile character of the PCCO/LAO heterostructure. In contrast to the general tendency reported for strained $\mathrm{La}_{0.5} \mathrm{Sr}_{0.5} \mathrm{CoO}_{3-\delta}$ (LSCO) films, we show that a nominal tensile strain can be also compatible with the presence of alternating $\mathrm{O}$ vacancy planes parallel to the interface, instead of perpendicular to it. That occurs thanks to the double cell of the film and the formation of the $(1 / 2,1 / 2,1 / 2)$ superstructure studied in this work. These results expand the possibilities of controlling interfacial physical properties via engineering of ordered oxygen-defect structures in strongly correlated oxides.
\end{abstract}

DOI: 10.1103/PhysRevMaterials.3.104407

\section{INTRODUCTION}

Cobalt oxides are attracting much attention in the condensed-matter community as they present a rich variety of interesting phenomena. Without being exhaustive, cobalt compounds present interesting properties for applications as mixed conductors, materials for solid-oxides fuel cells, thermopower applications, superconductivity, room-temperature (RT) ferromagnets, magnetoelectric materials, or materials for spintronics [1-4]. Spin-state transitions are among the most intriguing phenomena in solid-state physics, and cobalt oxides containing $\mathrm{Co}^{3+}$ ions have been the subject of continuing interest due to the possibility of Co ions to access different spin states by changing the temperature or the strain (using hydrostatic pressure or the substitution with smaller lanthanides). This ability to adopt different spin states is a key feature due to the relevance of the spin state of Co for electron mobility, the nature of magnetic coupling, or the structural stability. The perovskite structured cobaltites $\mathrm{LnCoO}_{3}, \mathrm{Ln}_{1-x} \mathrm{Ca}_{x} \mathrm{CoO}_{3}$ and $\mathrm{LnBaCo}_{2} \mathrm{O}_{5+d}(\mathrm{Ln}=$ lanthanide $)$ are very good examples of systems exhibiting spin state transitions, between low-spin (LS with $S=0$ and $t_{2 g}^{6}$ ) and higher-spin states (intermediate

\footnotetext{
*garcia.munoz@icmab.es
}

spin, IS, with $S=1, t_{2 g}^{5} e_{g}^{1}$; and high spin, HS, with $S=2$, $t_{2 g}^{4} e_{g}^{2}$ ), and associated with them, complex magnetic or orbital orders, giant magnetoresistance, unconventional metalinsulator transitions, etc. [5-9].

An interesting example is the half-doped cobaltite $\mathrm{Pr}_{0.50} \mathrm{Ca}_{0.50} \mathrm{CoO}_{3}$ (PCCO), with two $\mathrm{Co}$ ion types $\left(\mathrm{Co}^{3+}\right.$ and $\mathrm{Co}^{4+}$ in $1: 1$ ratio). It is metallic but undergoes a metal-insulator transition on cooling at $T_{\mathrm{MI}} \sim 80 \mathrm{~K}$, being insulating at low temperatures [10]. This compound does not present spontaneous magnetic order at low temperature, and early studies proposed that $\mathrm{Co}^{3+}$ ions could change from higher-spin states (IS or HS) above $T_{\mathrm{MI}}$ to a LS state below $T_{\mathrm{MI}}$. Later the spin-state change was experimentally proved [11]. Neutron-diffraction studies reveal that the structure is orthorhombic (Pnma crystal symmetry) and the volume unit cell contracts $(\sim 2 \%)$ during a structural transition across $T_{\mathrm{MI}}$ [12]. This shortening is the result of a $(\mathrm{Pr}, \mathrm{Ca})-\mathrm{O}$ bond distance contraction during the metal-insulator transition. Unexpectedly, it was also discovered that a $\operatorname{Pr}^{3+}$ to $\operatorname{Pr}^{4+}$ valence change accompanies the concurrent metal-insulator and spin-state transitions on cooling, producing transfer of electrons from Pr to Co sites [12-16]. This exceptional electronic mechanism in PCCO is based on the stabilization of the $\mathrm{Co}^{3+} \mathrm{LS}$ state at $T_{\mathrm{MI}}$, which forces the contraction of 
selected $\mathrm{Pr}-\mathrm{O}$ bonds and generates a first-order $\mathrm{Pr}^{3+}$ to $\mathrm{Pr}^{4+}$ valence shift below the transition. In addition, more recent experiments have shown the possibility of generating metallic domains in the insulating low-temperature phase of PCCO by ultrafast photoexcitation [17], making this material of interest in the area of ultrafast optical switching devices [17-21], and revealing that $\operatorname{Pr}_{0.5} \mathrm{Ca}_{0.5} \mathrm{CoO}_{3}$ heterostructures can present a great potential in photoresponse and photoelectric conversion applications [21].

Given the increasing importance of the oxides heterostructures for the design of artificial practical materials, in this paper we investigate the properties of epitaxial PCCO films by analogy with the exotic behavior found in ceramic PCCO samples. We report the fabrication and properties of epitaxial thin films of PCCO grown on $\mathrm{LaAlO}_{3}(001)$ (LAO) singlecrystalline substrate by pulsed-laser deposition (PLD). Oxygen vacancy formation in doped cobaltite films can be favored by increasing the strain produced by the substrate, as vacancy ordering acts as an effective mechanism for lattice mismatch accommodation. In this work the choice of the substrate was made with the aim of minimizing the mismatch with the cell dimensions of the PCCO perovskite.

\section{EXPERIMENTAL DETAILS}

First, PCCO was prepared in polycrystalline form following standard solid-state reaction methods, from the mixture of high-purity precursor oxides $\left(\mathrm{Co}_{3} \mathrm{O}_{4}, \mathrm{Pr}_{6} \mathrm{O}_{11}\right.$, and $\left.\mathrm{CaCO}_{3}\right)$. Several grindings, pressing, and firing steps were completed. The final sintering temperature was $1160^{\circ} \mathrm{C}$, in $\mathrm{O}_{2}$ atmosphere. In addition, the sample was treated under high oxygen pressure (at $900{ }^{\circ} \mathrm{C}$ and $P_{\mathrm{O} 2}=200$ bar during $14 \mathrm{~h}$, and at $475^{\circ} \mathrm{C}$ and $P_{\mathrm{O} 2}=150$ bar during $6 \mathrm{~h}$ ) in order to achieve an optimal oxygen content. Then, the polycrystalline target was used to fabricate epitaxial films of PCCO $(\sim 70 \mathrm{~nm})$, grown on (001)-oriented $\mathrm{LaAlO}_{3}$ (LAO) single-crystal substrates $\left(5 \times 5 \times 1 \mathrm{~mm}^{3}\right)$ by reflection high-energy electron diffraction assisted PLD technique, using a $\mathrm{KrF}$ excimer laser at energies of $65 \mathrm{~mJ}$ and a fluence of $1.4 \mathrm{~J} / \mathrm{cm}^{2}$ on a stoichiometric PCCO ceramic dense pellet as a target. After exploring different growth and annealing conditions, highquality epitaxial PCCO films were obtained at a substrate temperature $T_{\mathrm{S}}=700{ }^{\circ} \mathrm{C}$ carrying out the deposition in the presence of $P_{\mathrm{O} 2}=0.60 \mathrm{mbar}$ of $\mathrm{O}_{2}$ partial pressure. An annealing was done at $500{ }^{\circ} \mathrm{C}$ for $1 \mathrm{~h}$ under $P_{\mathrm{O} 2}=400 \mathrm{mbar}$, followed by a slow cooling to RT. Prior characterization included $\mathrm{x}$ ray, electron and atomic force microscopies, conventional dc magnetometry, and transport measurements. The films were characterized using conventional x-ray-diffraction (XRD) techniques $[\lambda(\mathrm{Cu} K \alpha)=1.5418 \AA]$ with a Siemens D-5000 diffractometer to measure $(\theta-2 \theta)$ scans, which provided information on film thickness, surface roughness, and lattice mismatch between substrate and film. The Bruker AXS D8 Advance equipped with an area detector (General Area Detector Diffraction System) was used to measure $\phi-2 \theta$ scans and image reciprocal-space maps recorded around the (103), (003), and (200) LAO reflections to check the presence of twinned film grains (unsuccessful) as well as complementary measurements of lattice alignments.
The direct current (dc) magnetization measurements, the field parallel to the film surface, were done using a superconducting quantum interferometer device from Quantum Design. The dc resistance $\rho(T)$ was measured by the four-probe method using a physical properties measurement system (Quantum Design). These transport measurements were made through metal-silver contacts grown on top of the surface film using a physical mask. The Ag was fixed onto the film doing a thermal treatment at $450^{\circ} \mathrm{C}$ for $1 \mathrm{~h}$ under $\mathrm{O}_{2}$ atmosphere. Contacts were done with an ultrasonic wire bonder (4526 Kulicke \& Soffa).

The surface quality of the films was imaged by means of atomic force microscopy (AFM) techniques using a scanning probe microscopy (Agilent 5100) system operating in dynamic mode at $174 \mathrm{kHz}$. In addition to conventional $\mathrm{x}$ ray measurements, the film structure was characterized at the surface diffraction beamline BM25B at the European synchrotron radiation facility (ESRF, Grenoble), using a one-dimensional detector ( $\mathrm{NaI}$ scintillator). The sample was mounted in a cryogenically adapted UHV baby chamber coupled to a six-circle diffractometer. The incident beam energy was set to $15.0 \mathrm{keV}[22,23]$. Moreover, the local crystal structure of PCCO films on LAO substrate was investigated employing a probe aberration-corrected JEOL ARM 200CF scanning transmission electron microscope (STEM) with a cold field-emission source operated at $200 \mathrm{kV}$, at Universidad Complutense de Madrid, Spain. The high-angle annular darkfield signal (80-180 mrad, HAADF) scales approximately with the square of the atomic number $Z$, and thus this imaging mode is referred to as $Z$-contrast imaging. In addition, due to the incoherent nature of the signal, $Z$-contrast images are directly interpretable and chemically sensitive. We also used STEM in combination with EELS to study the chemistry of the superstructure observed in the films, at the Center for Nanophase Materials Sciences (CNMS) at Oak Ridge National Laboratory (ORNL, USA). EELS spectrum images were acquired in a dedicated STEM, a Nion UltraSTEM, operated at $100 \mathrm{kV}$ and equipped with a fifth-order Nion aberration corrector and a Gatan Enfina EEL spectrometer. The principal component analysis (PCA) method was used to eliminate noise in atomic-resolution EELS elemental maps [24]. EELS elemental maps were generated by integrating the PCA-treated spectra over a 30-eV window, after background subtraction. The acquisition time was $0.03 \mathrm{~s}$ per pixel. We did not detect noticeable changes comparing films grown with and without postdeposition annealing in $\mathrm{O}_{2}$.

\section{EXPERIMENTAL RESULTS}

\section{A. Structural characterization}

Figure 1(a) shows a HAADF-STEM image of a 70-nmthick film deposited on (001)LAO. The film is continuous over long lateral length scale, with no significant roughness. As shown in Fig. S1 (Supplemental Material [25]) the film presents flat roughness, and AFM images show the presence of some disperse nanocrystalline grains with a height distribution between 5 and $20 \mathrm{~nm}$.

Although the lattice periodicity along the surface normal for both substrate and film are very close, they are not identical as observed in the insets of Fig. 1(b). In the $\theta-2 \theta$ diffraction patterns the PCCO films display (00l) reflections 
(a)

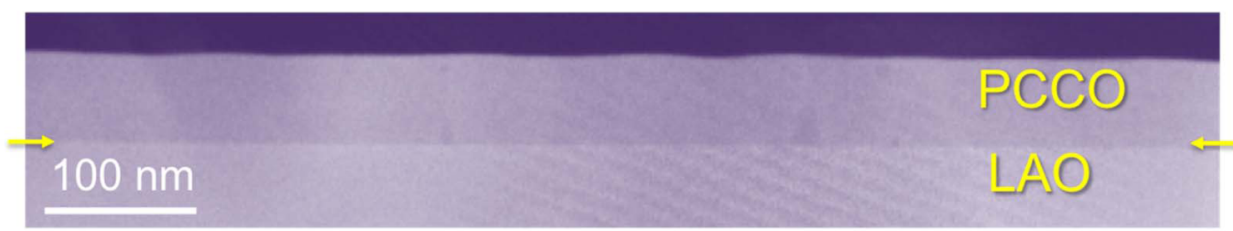

(b)

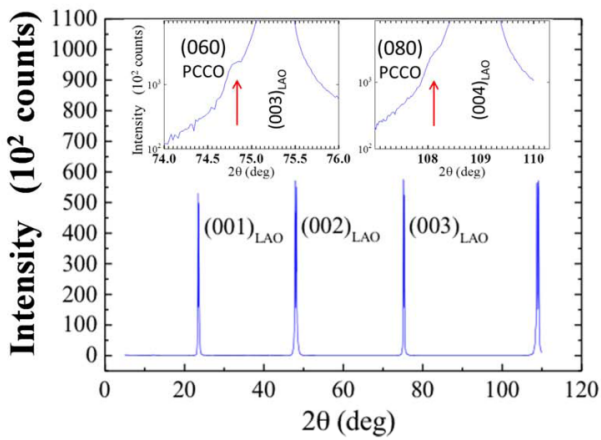

(c)

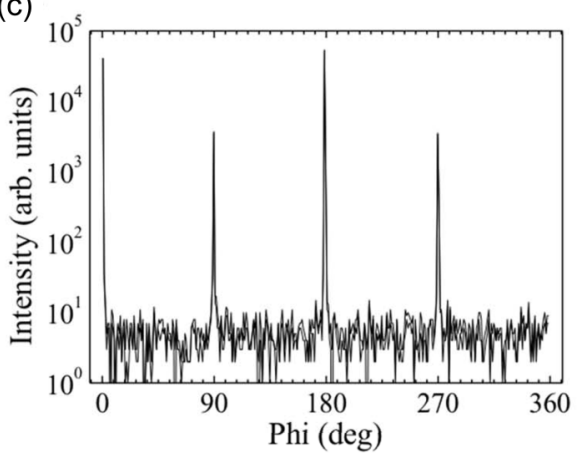

FIG. 1. (a) Low-magnification Z-contrast image of a 70-nm-thick film deposited on (001)LAO. (b) ( $\theta$-2 $\theta)$ x-ray-diffraction pattern for $\mathrm{Pr}_{0.50} \mathrm{Ca}_{0.50} \mathrm{CoO}_{3}$ on LAO. The insets show the (060) and (080) PCCO reflections close to the corresponding (00 $\ell$ ) LAO peaks (log scale). The main reflections are indexed in the pseudocubic LAO basis while film reflections are indexed according to the bulk PCCO crystal defined by the $\left(\sim \sqrt{ } 2 a_{0}, \sim 2 a_{0}, \sim \sqrt{ } 2 a_{0}\right)$ unit cell $\left(a_{0}\right.$, cubic perovskite). (c) Phi scan through the (202) PCCO reflection (taken at $2 \theta=32^{\circ}$ and $\left.\chi=45^{\circ}\right)$.

of the pseudocubic structure, which are more visible at high- $\theta$ angles, without signs of misorientation or impurities. The film grows with the long $b_{\text {Pnma }}$ axis perpendicular to substrate surface (out-of-plane orientation of PCCO perovskite films on the substrate). Figure 1(b) shows the $\theta-2 \theta$ XRD pattern for the $\mathrm{PCCO} / \mathrm{LAO}_{3}(001)$ system where the more intense peaks comes from the LAO substrate. The insets show the position of the (060) and (080) reflections from the PCCO film in comparison with, respectively, the positions of the (003) and (004) reflections from the LAO substrate. Azimuthal (phi) scans of film/substrate overlapping reflections separated by $90^{\circ}$ demonstrate a clear fourfold symmetry as observed in Fig. 1(c) for the (202) reflection. The lack of extra peaks denotes the absence of film-rotated domains not related to the LAO substrate symmetry. The out-of-plane lattice constants estimated for the substrate and the film from the position of $(00 l)$ peaks in $\theta-2 \theta$ XRD patterns [Fig. 1(b)] are $a^{\text {out }}$ LAO $[\exp ] \approx 3.788 \AA$ and $b_{\text {Pnma }}$ [exp] $\approx$ $7.616 \AA$. Reciprocal-space maps (RSM) around the (103), (002), and (003) pseudocubic reflections of the LAO substrate and PCCO thin film are shown in Fig. 2. A priori the nominal in-plane mismatch $\varepsilon=\left(a_{s}-a_{\mathrm{PCCO}}\right) / a_{s}$ is $\varepsilon=+0.41 \%$ (according to the single-crystal values: $a_{\mathrm{LAO}}=3.790 \AA$; $\left(a_{\text {Pnma }}, b_{\text {Pnma }}, c_{\text {Pnma }}\right)=(5.338,7.541,5.338) \AA$, Pnma cell of PCCO [12]). So, the relation of the lattice parameters between PCCO and the LAO substrate nominally should generate in-plane tensile stress, although very weak (much weaker than the one on LSAT, $+2.5 \%$, or STO, $+3.4 \%$ ).

From Fig. 2(b) we obtain the out-of-plane parameters $a_{\text {LAO }}^{\text {out }}[\exp ] \approx 3.789 \AA$ and $b_{\text {Pnma }}[\exp ] \approx 7.619 \AA$. The inplane lattice constants estimated for the substrate and the film from Fig. 2(a) are $a_{\text {LAO }}^{\text {in }}[\exp ] \approx 3.790 \AA$ and $a_{\text {Pnma }} \approx$
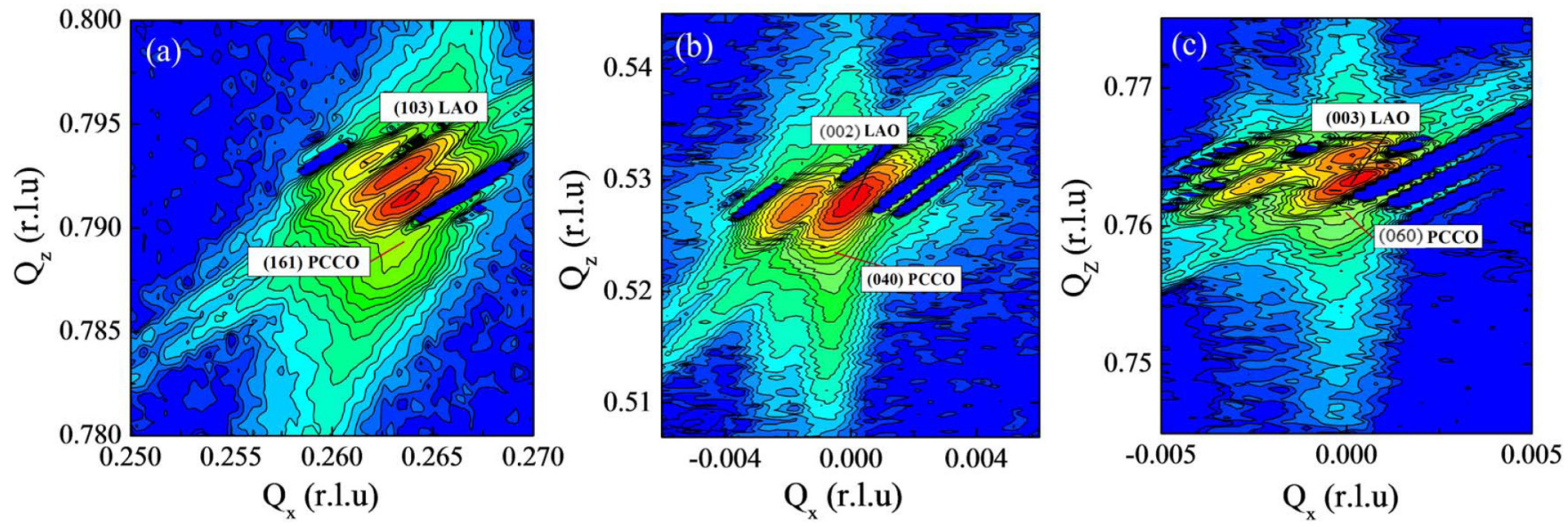

FIG. 2. Reciprocal-space maps around the substrate reflections (a) (103), (b) (002), and (c) (003) for the PCCO film grown on LAO substrate. LAO and film reflections are indexed in their respective $a_{\mathrm{LAO}}$ and $\left(a_{\mathrm{Pnma}}, b_{\mathrm{Pnma}}, c_{\mathrm{Pnma}}\right)$ basis. 

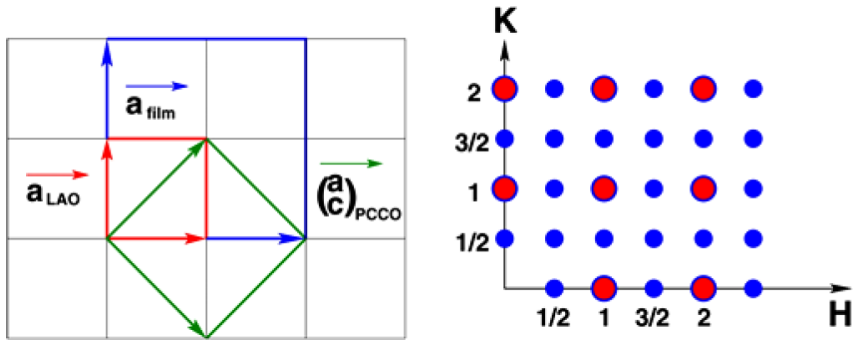

FIG. 3. Real (left) and reciprocal (right) in-plane projected space images of the PCCO/LAO (001) system. The left image shows the LAO basic cell defined by identical in-plane $a_{\text {LAO }}$ vectors (red), the bulk PCCO crystal with identical in-plane vectors, i.e., $\left(a_{\text {Pnma }}, c_{\text {Pnma }}\right)$ (green) and film unit cell with vectors $\left(a_{f}, b_{f}\right)=\left(2 a_{\mathrm{LAO}}, 2 a_{\mathrm{LAO}}\right)=$ $\left(\sqrt{ } 2 a_{\text {Pnma }}, \sqrt{ } 2 a_{\text {Pnma }}\right)$ (blue). The film unit cell out-of-plane vector $c_{\text {film }}=2 a_{\mathrm{LAO}}=b_{\mathrm{Pnma}}$. The reciprocal-space image (right) shows the set of reflections coming from the LAO and film cells. The filled red circles outlined in blue represent reflections having contributions from the LAO substrate and the film while blue filled circles show the observed set of reflections containing structural information from the film only. A similar set of reflections is generated at any $L=n / 2$ value with $n=1,2,3 \ldots$ integer.

$5.361 \AA\left(\approx \sqrt{ } 2 a^{\text {in }}{ }_{\text {LAO }}\right)$. For these estimations we have considered the most intense twin peak of LAO in the RSMs of Fig. 2 (the one labeled in the figures). The precision of the maximum for PCCO peaks is lower and in Fig. 2 the free end of the guide straight line that informs each PCCO reflection was taken as the reference position. Although it is somewhat difficult to assert a perfect alignment of the film and the substrate along $Q_{x}$ from Fig. 2, due to the error associated with the position of the PCCO maxima, the epitaxial film can be considered practically fully strained. This has been additionally corroborated by the synchrotron x-ray measurements shown in the next section, which show that the in-plane positions of film reflections are placed at half or integer values of those from the substrate.

\section{B. X-ray synchrotron diffraction study: Observation of a $P 2_{1} 2_{1} 2_{1}$ modulated structure}

The x-ray experimental results shown in the previous section confirm the epitaxial and in-plane strained PCCO film growth on the LAO substrate ("cube-on-cube" epitaxial relationship) with a nominal weak in-plane tensile stress of $\varepsilon=+0.41 \%$. The $\mathrm{x}$-ray characterization using conventional tools was enlarged with synchrotron diffraction measurements using the six-circle diffractometer of the surface diffraction beamline BM25B at the ESRF (Grenoble). The energy of the incoming $\mathrm{x}$-ray beam was set to $15.0 \mathrm{keV}$. The surface $\mathrm{x}$-ray-diffraction measurements revealed the presence of two different overlapped diffraction patterns from the PCCO film: the average bulk structure of the PCCO film, defined by cell lattice parameters $\boldsymbol{a}_{\text {Pnma }}, \boldsymbol{b}_{\text {Pnma }}, \boldsymbol{c}_{\text {Pnma }}$, and a set of reflections, as shown in Fig. 3, compatible with a film superstructure defined by lattice vectors:

$$
\left(\boldsymbol{a}_{f}, \boldsymbol{b}_{f}, \boldsymbol{c}_{f}\right)=\left(\begin{array}{ccc}
1 & 1 & 0 \\
1 & -1 & 0 \\
0 & 0 & 1
\end{array}\right)\left(\begin{array}{l}
\boldsymbol{c}_{\text {Pnma }} \\
\boldsymbol{a}_{\text {Pnma }} \\
\boldsymbol{b}_{\text {Pnma }}
\end{array}\right) .
$$

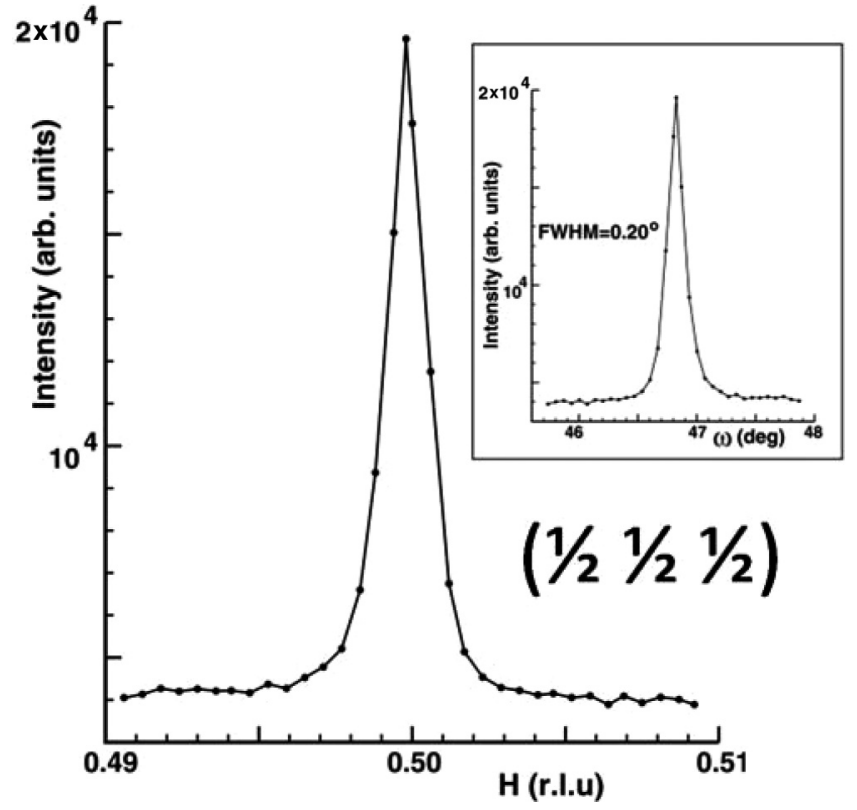

FIG. 4. Rocking curve corresponding to $(1 / 21 / 21 / 2)$ reflection of the PCCO film in reciprocal lattice units expressed in the LAO basis and degrees (inset).

This unexpected lattice defines a film unit cell where $\boldsymbol{a}_{f}$ and $\boldsymbol{b}_{f}$ vectors are placed on the surface plane (in plane) while $\boldsymbol{c}_{f}$ follows the LAO (001) out-of-plane surface direction. The three film lattice vectors are now parallel to the LAO axis (Fig. 3) and have periodicities that are two times those of the substrate [superstructure with associated wave vector $q=(1 / 2,1 / 2,1 / 2)]$. Such a structural modulation generates half fractional order reflections in the film along any of the three $(H, K, L)$ reciprocal-space directions, defined in the cubic basis of the LAO substrate, hereafter as produced by a film superstructure with lattice parameters $\left(a_{f}, b_{f}, c_{f}\right)=$ $\left(2 a_{\mathrm{LAO}}, 2 a_{\mathrm{LAO}}, 2 a_{\mathrm{LAO}}\right)=\left(\sqrt{ } 2 a_{\text {Pnma }}, \sqrt{ } 2 c_{\text {Pnma }}, b_{\text {Pnma }}\right)$.

Thereby, the rocking curve ( $\omega$ scan) of the $(1 / 21 / 21 / 2)$ reflection shown in Fig. 4 is a good example of the film lattice periodicity. Its full width at half maximum of $\Delta \omega=$ $0.20^{\circ}$ corresponds to a domain size of $\sim 100 \mathrm{~nm}$, which is indicative of the good crystallinity of the superstructure and the grown film. The excellent matching between LAO (001) substrate and the doubled cell of the film $\left[2 a_{\mathrm{LAO}} / a_{f}\left(=\sqrt{ } 2 a_{\mathrm{Pnma}}\right)=0.005 \%\right]$ favors this heteroepitaxial growth of the film on the LAO substrate. The double number of atoms allocated in this larger cell introduces structural degrees of freedom by lowering the symmetry of the space group with respect to the bulk one, as commented below, to favor the atomic arrangement of the Co octahedra in both the in-plane and out-of-plane dimensions. A detailed inspection of the film diffraction patterns shows crystal symmetry different from the Pnma space group characteristic of the PCCO bulk. Specific extinction rules present in bulk PCCO crystals, like those for $(0 K L),\left(\begin{array}{ll}H & 0 L\end{array}\right)$ and $(H K 0)$ reflections, are no longer put forward in the film. The only experimentally confirmed extinctions correspond to the $\left(\begin{array}{ll}H & 0\end{array}\right),\left(\begin{array}{ll}0 & K\end{array}\right)$, and $\left(00 \mathrm{~L}\right.$ ) family of reflections, in agreement with the $P 2_{1} 2_{1} 2_{1}$ (No. 19) space-group symmetry. 

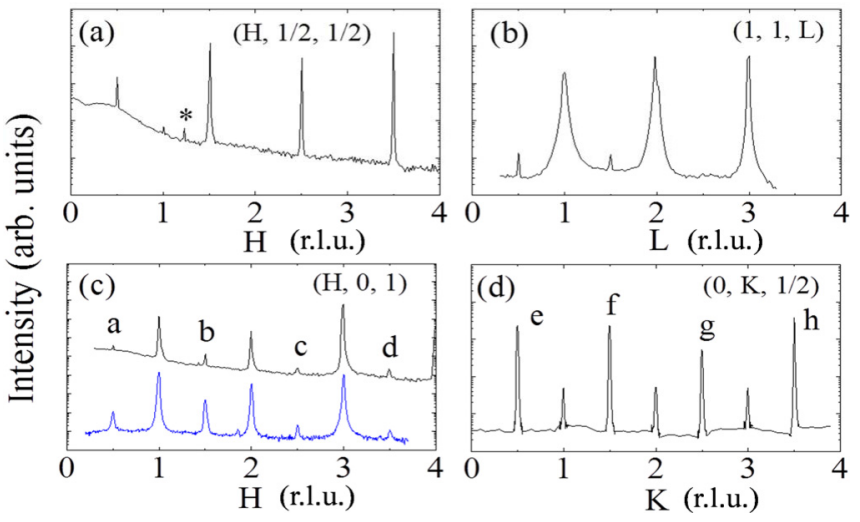

FIG. 5. Selected synchrotron x-ray-diffraction scans at RT from PCCO thin film. (*) marks an impurity peak. Selected $(H, K, L)$ reflections in (c), (d), defined in the basis of the PCCO film, correspond to: $a=(1,0,2), b=(3,0,2), c=(5,0,2), d=(7,0,2)$, $e=(0,2,1), f=(0,4,1)$, and $g=(0,6,1)$, respectively. Plots are in log scale. In (c) the blue curve shows the data from the measurement identical to the black one (RT) but collected at $68 \mathrm{~K}$.

The presence of different types of PCCO orientational domains related with their bulk $a_{\mathrm{Pnma}}$ and $c_{\mathrm{Pnma}}$ lattice constants have been disregarded due to the absence of characteristic reflections associated with these periodicities in the experimental diffraction pattern. That is, in case of structures with $b_{\text {Pnma }}$ lattice vector lying on the surface it would be aligned along one of the in-plane LAO direction periodicities due to its low lattice mismatch $\left(b_{\mathrm{Pnma}} \approx 2 a_{\mathrm{LAO}}\right)$. However, in such case $a_{\text {Pnma }}$ or $c_{\text {Pnma }}$ lattice vectors would not be commensurate with those of the LAO substrate ( $a_{\mathrm{Pnma}}$ and $c_{\mathrm{Pnma}}$ periodicities differ from those of $a_{\mathrm{LAO}}$ or $\left.2 a_{\mathrm{LAO}}\right)$. Therefore, in that situation, reflections with periodicities $\left(a_{\mathrm{LAO}} / a_{\mathrm{Pnma}}\right.$ or $\left.c_{\mathrm{Pnma}}\right)=$ 0.71 reciprocal lattice units) along $H$ or $L$ reciprocal-space LAO directions would have been observed but they were not, or are absolutely residual.

Figure 5 shows some measured directional $(H, K, L)$ scans for several families of film reflections. Reflections with all three $(H K L)$ indexes different from zero are permitted [Figs. 5(a) and 5(b)]. However, looking at the reflections of the type $(H 0 L)$ and $(0 K L)$, we observe the presence of extra peaks labeled " $a$," " $b$," "c," and " $d$ " in Fig. 5(c), and " $e, " ~ " f, " ~ " g$," and " $h$ " in Fig. 5(d), respectively, that are not permitted in the crystalline structure of bulk PCCO. The presence of these reflections rules out the Pnma symmetry in the film as well as any of the equivalent settings to this space group corresponding to different orientations of the $\left(a_{\text {Pnma }}, b_{\text {Pnma }}, c_{\text {Pnma }}\right)$ lattice vectors relative to those of the LAO substrate: Pmcn, Pmnb, Pcmn, Pnam, or Pbnm.

\section{Low-temperature behavior}

For comparison with the exotic metal-insulator and valence transitions in bulk PCCO under cooling, the PCCO/LAO film was characterized at low temperatures by means of structural synchrotron XRD, magnetic, and transport measurements. Possible changes in the film structure were investigated after cooling the film down to $68 \mathrm{~K}$. At this temperature some selected synchrotron $\mathrm{x}$-ray-diffraction scans were recorded.
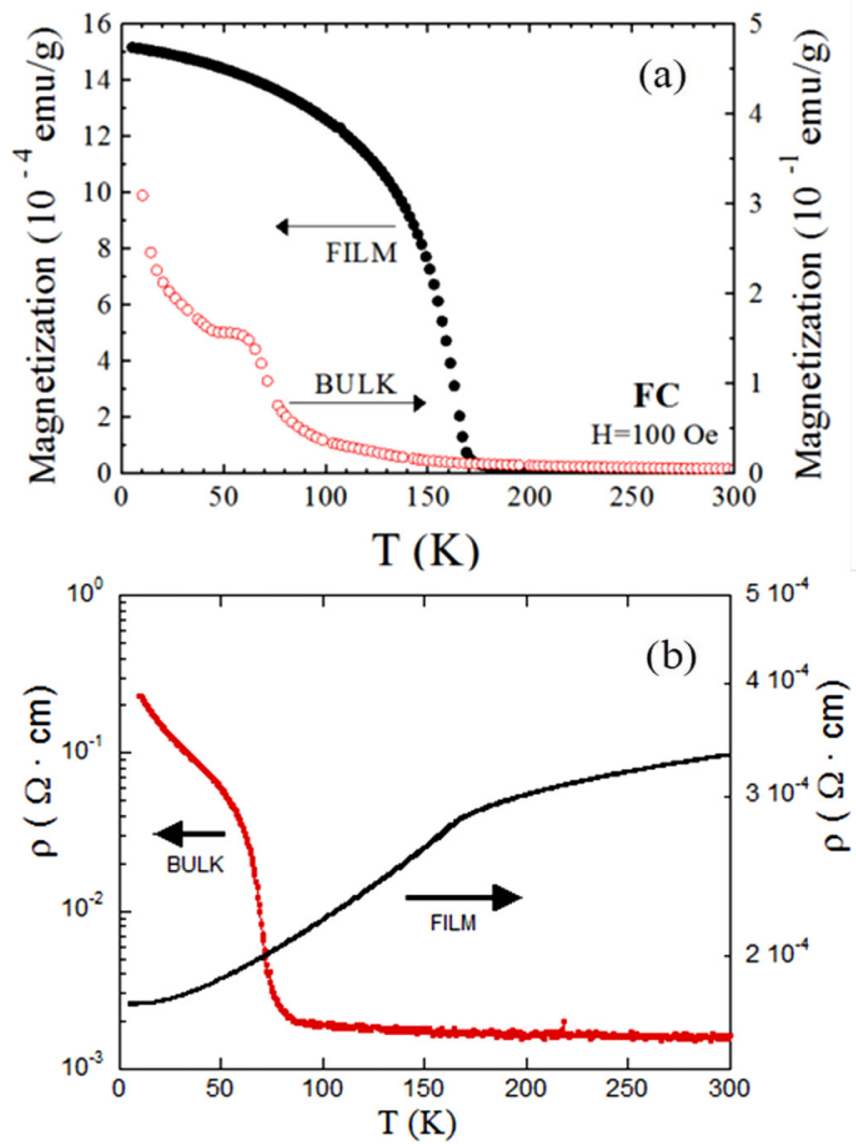

FIG. 6. (a) Magnetic susceptibility of the PCCO/LAO film (left axis), comparison with that in the PCCO bulk sample (target) (right axis). Field-cooled curves measured on heating under 100 Oe. (b) Comparison of the zero-field resistivities measured in the film and the bulk sample.

It was confirmed that the reflection conditions found at RT remain at low temperature. Moreover, slightly higher intensities compared to RT were detected at low temperatures when comparing identical reflections. For comparison, in Fig. 5(c) we show two identical directional scans collected at $300 \mathrm{~K}$ (black) and $68 \mathrm{~K}$ (blue) confirming the persistence of the $P 2_{1} 2_{1} 2_{1}$ symmetry and very similar relative distribution of intensities.

The magnetic and conductivity properties of the film also confirmed the suppression of the singular structural and metalinsulator transition reported in stoichiometric ceramic PCCO samples. Figure 6(a) shows the temperature dependence of the magnetic susceptibility of the PCCO film (left axis) in the 2-300 K range, as measured under field cooling and using a dc field of 100 Oe. For comparison, the $\chi(T)$ plot for the reference bulk sample measured in the same conditions is shown in the same figure (right axis). In the bulk system a peaklike anomaly or shoulder in the magnetic susceptibility near 70-80 K gives evidence of the concurrent MI and abrupt spin transition. $\mathrm{Co}^{3+}$ sites abruptly change their spin state from HS to LS under cooling through $T_{\mathrm{MI}}$. As a result, there is no spontaneous ferromagnetism (FM) at low temperature but it can be induced under field by polarization of the $\mathrm{Co}^{4+}$ 
atomic moments. This is in contrast with the film, whose magnetization unveils the presence of FM order below $T_{\mathrm{C}}=$ $170 \mathrm{~K}$. The corresponding $\rho(T)$ curve in Fig. 6(b) discloses the metallic character of the film in the whole temperature range, and the rise of the conductivity due to the polarization of Co spins below $T_{\mathrm{C}}$. So, Fig. 6(b) clearly shows how the upturn in the resistivity curve of PCCO bulk has been suppressed in the PCCO/LAO film with $P 2{ }_{1} 2{ }_{1} 2_{1}$ symmetry, which remains metallic and FM at low temperatures.

\section{STEM}

Figure 7(a) shows a high-resolution $Z$-contrast image of the interface region, showing a sharp and coherent $\mathrm{PCCO} / \mathrm{LAO}$ interface, free of misfit dislocations, that confirms the cube-on-cube epitaxy. A closer examination exposes a superstructure in the PCCO film, as shown in more detail in the inset, where every other $\mathrm{CoO}_{2}$ plane presents a significant lower contrast. The Fourier transform of this image (see the inset) shows superlattice peaks in between the primary diffraction spots, thus confirming the presence of the superstructure. The contrast observed in our images is well known to be due to the structural relaxations that result from the ordering of oxygen vacancies [26-28]. As has been reported, the dimmer/brighter pattern of Co-O planes stems from a modulation in cation spacing resulting from the $\mathrm{O}$ content modulation [26], not from the variation in $\mathrm{O}$ content directly. Figure 7(b) shows the histogram of the $\mathrm{Pr} / \mathrm{Ca}-\mathrm{Pr} / \mathrm{Ca}$ distance $(\Delta z$, out of plane) between successive $\mathrm{Pr} / \mathrm{Ca}$ planes parallel to the interface in the region of the image occupied by the film. Two characteristic distances are easily identified in the histogram, $d_{1}$ and $d_{2}$, which appear also indicated in Fig. 7(a). Figures 7(a) and 7(b) show that dimmer stripes in the $Z$-contrast image correspond to the dilated $\mathrm{Pr} / \mathrm{Ca}-\mathrm{Pr} / \mathrm{Ca}$ distance $d_{2}\left(d_{2} \sim 3.974\right.$ vs $\left.d_{1} \sim 3.667 \AA\right)$. The columns in the histogram that give rise to the third distance $\left(d_{\mathrm{La}-\mathrm{La}} \sim\right.$ $3.792 \AA$ ) in Fig. 7(b) proceed from the region in the $Z$-contrast image of Fig. 7(a) occupied by the substrate $\left(\mathrm{LaAlO}_{3}\right)$. Therefore, the $d_{\mathrm{La}-\mathrm{La}}$ distance corresponds to the separation between successive La planes in the substrate (La-La $\Delta z$ distance). Accordingly, the observed out-of-plane lattice parameter matches perfectly well the value expected in the LAO (3.790 $\AA$ ). The panels of Fig. 8 show the atomic resolution maps of the $\mathrm{O} K$, Co $L_{2,3}, \operatorname{Pr} M_{4,5}$, and $\mathrm{Ca} L_{2,3}$ edges, around $530,779,931$, and $346 \mathrm{eV}$, respectively. Yellow arrows mark the dimmer Co-O planes, where the $\mathrm{O} K$ signal decreases and signals the presence of $\mathrm{O}$ vacancies. In addition, the cations nearest to the vacancies are somewhat displaced, making the Co $L$-edge signal decrease slightly in these positions. This effect is even experimentally more evident in the HAADFSTEM images of our film. Pr $M$ - and Ca $L$ elemental maps show the structural relaxation stemming from the presence of the $\mathrm{O}$ vacancies, which promote the $\mathrm{Pr} / \mathrm{Ca}$ planes to move further apart from the Co-O plane $\left(d_{2}>d_{1}\right.$ in the Pr- and Ca maps of Fig. 8). To illustrate better these effects we show as an example the sketch of a brownmillerite structure $\left[A B \mathrm{O}_{2.5}\right]$ in Fig. 8, where these effects are more pronounced due to a larger amount of ordered $\mathrm{O}$ vacancies: besides the difference between $d_{1}$ and $d_{2}$, the single positions in, respectively, the metal and oxygen columns split in more than one position along (a)

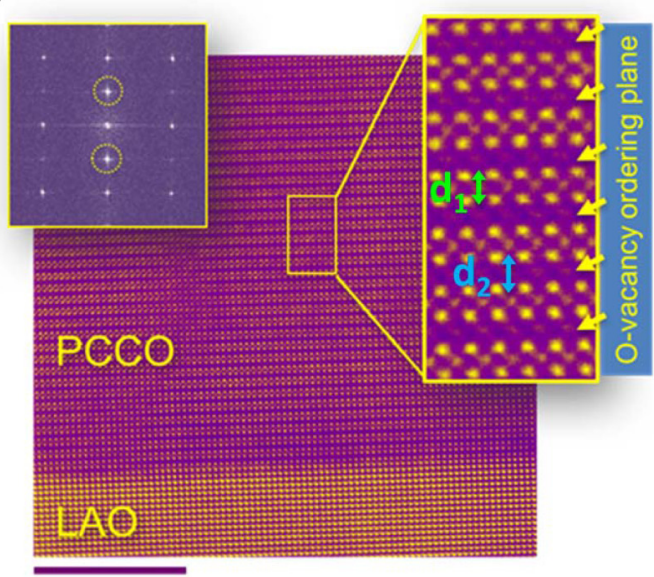

(b)

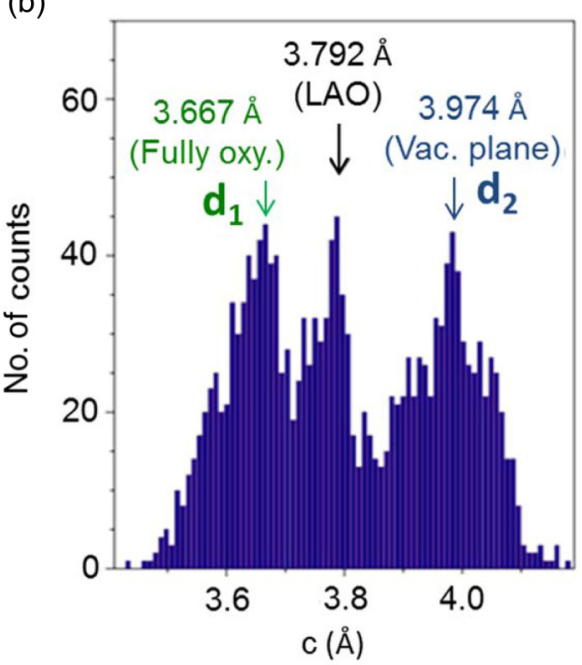

FIG. 7. (a) High-resolution $Z$-contrast image of the PCCO/LAO interface. As the intensity on the image approximately scales with $Z^{2}$, so the brighter atomic columns correspond to the $\mathrm{Pr} / \mathrm{Ca}$ cations and the dimmer ones to Co. Lighter atoms are not visible. $d_{1}$ and $d_{2}$ signal different $(\mathrm{Pr} / \mathrm{Ca})-(\mathrm{Pr} / \mathrm{Ca})$ distances. The inset on the left shows the image Fourier transform. Yellow circles mark two superlattice peaks. The inset on the right shows a magnified area of the film. The arrows mark the O-deficient planes. Scale bar $10 \mathrm{~nm}$. (b) Histogram of the out-of-plane distance values $(\Delta z)$ between lanthanide neighbors in successive layers of the structure obtained from the image in (a). Three peaks are clearly observed: the one corresponding to the lattice parameter of the LAO substrate (black arrow, La-La distance); the $(\mathrm{Pr} / \mathrm{Ca})-(\mathrm{Pr} / \mathrm{Ca})$ distance from the PCCO stoichiometric planes (green arrow, $d_{1}$ ), and the analog lattice distance corresponding to the O-deficient $\mathrm{CoO}_{x}$ planes (blue arrow, $d_{2}$ ).

the columns-perpendicular to the plane image-containing the $\mathrm{O}$ vacancies (atomic displacements induced by the vacancies).

\section{DISCUSSION}

$\operatorname{Pr}_{0.5} \mathrm{Ca}_{0.5} \mathrm{CoO}_{3}$ cobaltite exhibits exotic properties and a metal-insulator transition, being considered a "strongly correlated spin-crossover" system. PCCO is one of the first 

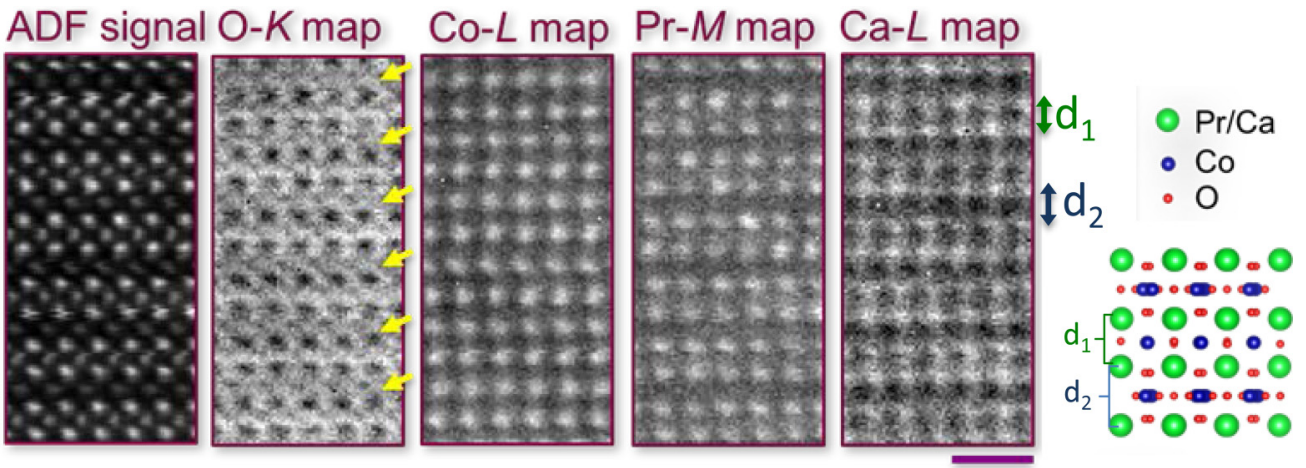

FIG. 8. From left to right, annular dark field (ADF) signal and elemental maps corresponding to the O $K$, Co $L_{2,3}$, $\operatorname{Pr} M_{4,5}$, and Ca $L_{2,3}$ edges. Integration windows $30 \mathrm{eV}$ wide were used after background subtraction using a power-law fit. Scale bar $1 \mathrm{~nm}$. For comparison, the sketch corresponds to a brownmillerite structure $\left(\mathrm{PCCO}_{2.5}\right.$, see explanation in the text).

strongly correlated oxides able to offer a large and ultrafast photoresponse in the insulating state. As reported by Okimoto et al., metallic domains can be induced by laser stimulation [17], and hence the implementation of the insulating state of PCCO in heterostructures presents a great interest for photoresponse and photoelectric conversion applications. Singlecrystalline epitaxial films of PCCO have been fabricated on LAO (100) substrates by means of pulsed-laser deposition. This substrate was selected with the aim of minimizing the mismatch with the film. Reducing the mismatch, we have tried to minimize $\mathrm{O}$ vacancy formation avoiding the need to compensate strong tensile or compressive strains through anisotropic vacancy distributions [27]. We have obtained and characterized epitaxial films $(70 \mathrm{~nm})$ with a very weak inplane stress $(\varepsilon=+0.41 \%$, tensile). The films (annealed under oxygen during the last stage of the fabrication) grow epitaxially on the surface with two times the substrate periodicity $\left(2 a_{0} \times 2 a_{0} \times 2 a_{0}\right)$ and with the long $b$ axis of the Pnma bulk perovskite perpendicular to it. Synchrotron diffraction reveals that the big number of atoms present in the reconstructed cell increases the degrees of freedom for relaxing the structure which is equivalent to lowering its symmetry: It presents a symmetry reduction from the space group Pnma (No. 62) to $P 22_{1} 2_{1}$ (No. 19). The films are metallic and ferromagnetic with $T_{\mathrm{C}}=170 \mathrm{~K}$ indicating that $\mathrm{Co}^{3+}$ ions are in a higher spin state than in bulk fully stoichiometric PCCO, which does not show spontaneous magnetic order. The proportion of excited $\mathrm{Co}^{3+}$ spin states (IS or HS) therefore is higher in the film. X-ray synchrotron investigations decreasing temperature down to $80 \mathrm{~K}$ did not reveal structural modifications at low temperature, neither in the $P 2{ }_{1} 2_{1} 2_{1}$ symmetry of the film nor in the distribution of diffracted intensity. Experimentally we did not detect any sign of the first-order phase transition in the polycrystalline bulk, confirming the suppression of the Co spin-state, valence, and metal-insulator concurrent transitions. On this basis, next we will analyze some relevant aspects related to the features observed in these films with very little tensile strain.

In general, strain affects bond lengths and angles and the stability of the cobalt spin states through the modification of the crystal-field energy term. On one hand, a compressive in-plane strain acts, increasing the Co-O-Co distortion in the plane and favoring the insulating state. In $\mathrm{Pr}-\mathrm{Ca}-\mathrm{Co}-\mathrm{O}_{3}$ and $(\mathrm{Pr}, \mathrm{Y})-\mathrm{Ca}-\mathrm{Co}-\mathrm{O}_{3}$ perovskites, an additional effect is at play because this strain is also favoring the charge transfer from $\operatorname{Pr}^{3+}$ (giving $\left.\mathrm{Pr}^{4+}\right)$ to $\mathrm{LS} \mathrm{Co}{ }^{4+}$ cations $\left(3 d t_{2 g}^{5} e_{g}^{0}, S=\right.$ $1 / 2)$, transforming to $\left.\mathrm{LS} \mathrm{Co}^{3+}\left[3 d t_{2 g}^{6} e_{g}^{0}, S=0\right]\right)[11,13]$. In this way, $\left(\operatorname{Pr}_{1-y} \mathrm{Y}_{y}\right)_{0.7} \mathrm{Ca}_{0.3} \mathrm{CoO}_{3}$ epitaxial films $(y=0.125)$ grown on the $\mathrm{SrLaAlO}_{4}$ substrate $(\varepsilon=-0.5 \%$, in-plane compressive stress) showed a semiconducting behavior in the whole temperature range, and a smooth anomaly in the resistivity $\rho(T)$ was interpreted as the reminiscent signature of the simultaneous $\mathrm{Pr}^{3+} / \mathrm{Pr}^{4+}$ valence change and metalinsulator transition reported in the bulk $[29,30]$. In bulk PCCO the transition involves a strong hybridization between $\operatorname{Pr} 4 f$ and $\mathrm{O}_{\text {ap }} 2 p$ orbitals in the $\mathrm{PrO}_{2}$ planes [11,12] $\left(\mathrm{O}_{\mathrm{ap}}\right.$ : apical oxygens in $\mathrm{CoO}_{6}$ octahedra, located in $\mathrm{PrO}_{2}$ layers).

On the other hand, it is well known that these systems can easily develop other mechanisms distinct to the simple modification of bond lengths and angles to release the mismatch $[27,28]$. Thus, the promotion of oxygen vacancy ordering to favor lattice mismatch accommodation was confirmed in epitaxial $\mathrm{La}_{0.5} \mathrm{Sr}_{0.5} \mathrm{CoO}_{3-\delta}$ (LSCO) thin films [27]. Other mechanisms may also occur, as observed in strained ferrimagnetic $\mathrm{LnCoO}_{3}$ perovskite films (e.g., for $\varepsilon=+1.7 \%$ on LSAT) where the in-plane modulation components are determined by a spin-orbital superstructure [31].

In the context of oxygen vacancy promotion to favor lattice accommodation, we will show below that our results do not entirely follow the general model for strain relief in cobaltites and tensile substrates. Let us recall first that Ref. [26] reported how lattice mismatch in epitaxial LSCO films can be used to manipulate the direction of the modulation vector associated with the long-range vacancy order: (a) in tensile-strained LSCO thin films [e.g., on $\operatorname{STO}(001), \varepsilon=+1.8 \%$ ] the modulation vector $q=1 / 2$ is parallel to the interface (in plane), but (b) it is perpendicular to it [out of plane, as in Fig. 7(a)] in compressive-strained LSCO films [e.g., on $\mathrm{LAO}(001), \varepsilon=$ $-1.3 \%$ ] [27,32]. Our observations indicate that the $\mathrm{O}$ vacancy superstructure provides the primary mechanism of lattice mismatch accommodation also in PCCO films. However, in contrast to the usual observations or general rules in strained LSCO films [27], the modulation observed in the Z-contrast STEM images of Fig. 7(a) for PCCO is perpendicular to the interface. This is so in spite of the tensile effect done by the LAO substrate. Hence, at first glance, the present half-doped 
PCCO/LAO cobaltite films seem to differ from the general behavior previously drawn in half-doped LSCO films. A better understanding is required because the simple assumed model that strain relief in the case of a tensile substrate occurs through $\mathrm{O}$ vacancy planes stacking perpendicular to the interface to enable in-plane expansion does not explain our observations.

We have shown that the excellent matching between LAO (001) substrate and the doubled cell of the film determines the particular heteroepitaxial growth of the film on the LAO substrate. The in-plane area of the film unit cell doubles $\left(a_{f}=\right.$ $\left.b_{f}=2 a_{\mathrm{LAO}}\right)$ and its associated structural relaxation favors a brownmilleritelike superstructure with a well-defined orientation of the $\mathrm{O}$ vacancy planes parallel to the interface. Concurrent out-of-plane $(0,0,1 / 2)$ and in-plane $(1 / 2,1 / 2,0)$ modulations of different nature are superposed. On one hand, vacancy planes produce the $(0,0,1 / 2)$ modulation in the distance between $\mathrm{Pr} / \mathrm{Ca}$ and $\mathrm{Co}-\mathrm{O}$ planes observed in the $Z$-contrast images. On the other hand, the $\mathrm{O}$ vacancy planes parallel to the interface also induce in-plane atomic displacements on the atoms nearest to the vacancies (such as Co and $\mathrm{O}$ ions) which are favored and easily permitted in the double cell $\left(2 a_{\mathrm{LAO}}, 2 a_{\mathrm{LAO}}, c_{f}\right)$ of the PCCO film. The final result is the $(1 / 2,1 / 2,1 / 2)$ superstructure here unveiled by synchrotron $\mathrm{x}$ ray.

Therefore, the $\mathrm{O}$ vacancy planes solely parallel to the interface are favored in the PCCO/LAO system thanks to the perfect matching of the doubled cell of the film with the substrate. This also explains why the $\mathbf{q}=(1 / 2,1 / 2,1 / 2)$ superstructure does not occur in analogous PSCO/LAO films (PSCO: $\mathrm{Pr}_{0.5} \mathrm{Sr}_{0.5} \mathrm{CoO}_{3}$ ), prepared under similar conditions and presenting in-plane compressive strain of $\varepsilon=-1.1 \%$ [33]. The observations in PCCO/LAO are at odd with the standard models to anticipate the orientation of $\mathrm{O}$ vacancy ordering in similar films (such as LSCO) from the relative dimensions of the substrate.

A remarkable finding was that in bulk PYCCO $\left[\left(\mathrm{Pr}_{0.85} \mathrm{Y}_{0.15}\right)_{0.7} \mathrm{Ca}_{0.3} \mathrm{CoO}_{3-\delta}\right]$ the charge-transfer transition between Pr and Co produces a fully reversible order/disorder transition of the $\mathrm{O}$ vacancies at cryogenic temperatures $\left(T_{\mathrm{MI}} \sim 165 \mathrm{~K}\right)$ [34]. This fact agrees with a random distribution of the $\mathrm{Pr}^{4+}-\mathrm{O}_{\mathrm{ap}}$ bonds in the insulating phase. Moreover, it emphasizes the relationship of competence between the superstructure (ordered) and the charge-transfer (insulating, with intrinsic disorder) phases. In the films the atomic displacements that accompany the $P 2{ }_{1} 2{ }_{1} 2_{1}$ symmetry could shorten or lengthen $\mathrm{Pr}-\mathrm{O}_{\mathrm{ap}}$ bonds in some $\mathrm{PrO}_{2}$ layers of the superstructure but these details are not known.

\section{CONCLUSION}

The properties of epitaxial PCCO films grown on $\mathrm{LAO}(001)$ have been investigated by analogy with the exotic behavior of PCCO ceramic samples. The substrate was selected with the aim of minimizing the mismatch with the cell dimensions of the PCCO perovskite. The films (annealed under 400 mbar $\mathrm{O}_{2}$ partial pressure) grow with $\varepsilon=+0.41 \%$ tensile in-plane stress. The too-simplistic model for a tensile substrate stating that strain relief for cobaltites occurs through $\mathrm{O}$ vacancy planes stacking perpendicular to the interface (modulation $q=1 / 2$ parallel to the plane of the film) is not verified in the present case. The $\mathbf{q}$ modulation observed in $\mathrm{PCCO} / \mathrm{LAO}$ is of $(0,0,1 / 2)$ type. But in addition, we have established that (in these films with reduced mismatch) the structure relaxes through a second modulation $(1 / 2,1 / 2,0)$ that doubles the in-plane area of the actual unit cell. As a result a $\mathbf{q}=(1 / 2,1 / 2,1 / 2)$ superstructure governs the atomic displacements generating a loss of symmetry elements and the final $P 2_{1} 2_{1} 2_{1}$ symmetry. This superstructure does not occur in analogous PSCO/LAO films, prepared under similar conditions and presenting in-plane compressive strain.

The films are metallic and ferromagnetic $\left(T_{\mathrm{C}}=170 \mathrm{~K}\right)$, and neither the electrical resistivity, nor the magnetic or structural evolutions show reminiscent signs of the $\operatorname{Pr}^{3+}$ to $\mathrm{Pr}^{4+}$ valence transition. Our observations bear out that further experimental efforts are needed to improve our understanding of the mechanisms able to inhibit (/activate) the metal-insulator transition in $\mathrm{Pr}-\mathrm{Ca}-\mathrm{Co}-\mathrm{O}$ films. Apart from the density of $\mathrm{O}$ vacancies, the crystallization of superstructures from vacancy ordering can be crucial through two different mechanisms. First, the atomic displacements producing the supercell modify the interatomic distances and can affect the $\mathrm{Pr}-\mathrm{O}_{\mathrm{ap}}$ bond lengths in the $\mathrm{PrO}_{2}$ planes. Second, the required strong hybridization of a number of $\operatorname{Pr}(4 f)-\mathrm{O}_{\mathrm{ap}}(2 p)$ pairs ( $\mathrm{Pr}$ is randomly distributed in the A site) requires generating an extra disorder in the structure [34], which most probably competes with the ordering promoted by the $(1 / 2,1 / 2,1 / 2)$ superstructure.

\section{ACKNOWLEDGMENTS}

We acknowledge financial support from the Spanish Ministerio de Ciencia, Innovación y Universidades (MICINN), through Projects No. MAT2015-686760-02-2-P, No. RTI2018-098537-B-C21, and No. RTI2018-098537-BC22, and "Severo Ochoa" Programme for Centres of Excellence in R\&D (Grant No. SEV-2015-0496). The former are cofunded by ERDF of European Union, as Project No. EFA194/16 TNSI (POCTEFA/UE-FEDER). We thank also the Spanish MICINN and the Consejo Superior de Investigaciones Científicas for financial support and for provision of synchrotron radiation facilities (Grant No. CRG-BM25B, ESRF). J.G. acknowledges the Ramon y Cajal program (Grant No. RYC-2012-11709). Research at ORNL (STEM-EELS) was supported by the US Department of Energy (DOE), Basic Energy Sciences (BES), Materials Sciences and Engineering Division (SJP and MV).
[1] Z. Shao and S. M. Haile, Nature (London) 431, 170 (2004).

[2] Y. Wang, N. S. Rogado, R. J. Cava, and N. P. Ong, Nature (London) 423, 425 (2003).
[3] W. Kobayashi, S. Ishiwata, I. Terasaki, M. Takano, I. Grigoraviciute, H. Yamauchi, and M. Karppinen, Phys. Rev. B 72, 104408 (2005) 
[4] K. Takada, H. Sakurai, E. Takyama-Muromachi, F. Izumi, R. A. Dilanian, and T. Sasaki, Nature (London) 422, 53 (2003).

[5] M. A. Señarís-Rodríguez and J. B. Goodenough, J. Solid State Chem. 116, 224 (1995).

[6] T. Saitoh, T. Mizokawa, A. Fujimori, M. Abbate, Y. Takeda, and M. Takano, Phys. Rev. B 55, 4257 (1997).

[7] I. O. Troyanchuk, N. V. Kasper, D. D. Khalyavin, H. Szymczak, R. Szymczak, and M. Baran, Phys. Rev. Lett. 80, 3380, (1998).

[8] C. Frontera, J. L. García-Muñoz, A. Llobet, and M. A. G. Aranda, Phys. Rev. B 65, 180405(R) (2002).

[9] A. Maignan, V. Caignaert, B. Raveau, D. Khomskii, and G. Sawatzky, Phys. Rev. Lett. 93, 026401 (2004).

[10] S. Tsubouchi, T. Kyômen, M. Itoh, P. Ganguly, M. Oguni, Y. Shimojo, Y. Morii, and Y. Ishii, Phys. Rev. B 66, 052418 (2002).

[11] J. Herrero-Martín, J. L. García-Muñoz, K. Kvashnina, E. Gallo, G. Subías, J. A. Alonso, and A. J. Barón-González, Phys. Rev. B 86, 125106 (2012).

[12] A. J. Barón-González, C. Frontera, J. L. García-Muñoz, J. Blasco, and C. Ritter, Phys. Rev. B 81, 054427 (2010).

[13] J. Hejtmánek, E. Šantavá, K. Knížek, M. Maryško, Z. Jirák, T. Naito, H. Sasaki, and H. Fujishiro, Phys. Rev. B 82, 165107 (2010).

[14] K. Knížek, J. Hetjmánek, P. Novák, and Z. Jirák, Phys. Rev. B 81, 155113 (2010).

[15] J. L. García-Muñoz, C. Frontera, A. J. Barón-González, S. Valencia, J. Blasco, R. Feyerherm, E. Dudzik, R. Abrudan, and F. Radu, Phys. Rev. B 84, 045104 (2011).

[16] J. Herrero-Martín, J. L. García-Muñoz, S. Valencia, C. Frontera, J. Blasco, A. J. Barón-González, G. Subías, R. Abrudan, F. Radu, E. Dudzik, and R. Feyerherm, Phys. Rev. B 84, 115131 (2011).

[17] Y. Okimoto, X. Peng, M. Tamura, T. Morita, K. Onda, T. Ishikawa, S. Koshihara, N. Todoroki, T. Kyomen, and M. Itoh, Phys. Rev. Lett. 103, 027402 (2009).

[18] W. Koshibae, N. Furukawa, and N. Nagaosa, Phys. Rev. B 87, 165126 (2013).

[19] Y. Kanamori, H. Matsueda, and S. Ishihara, Phys. Rev. Lett. 107, 167403 (2011)

[20] K. Seko, Y. Okimoto, M. Kurashima, R. Fukaya, T. Egawa, T. Ishikawa, K. Onda, S. Y. Koshihara, T. Kyomen, and M. Itoh, Acta Phys. Pol., A 121, 369 (2012).
[21] S. G. Zhao, A. Gu, X. L. Yan, L. M. Hao, Y. Xie, T. Zhang, and K. X. Jin, Eur. Phys. Lett. 108, 67007 (2014).

[22] J. Rubio-Zuazo, P. Ferrer, A. López, A. Gutiérrez-León, I. da Silva, and G. R. Castro, Nucl. Instrum. Methods Phys. Res., Sect. A 716, 23 (2013).

[23] J. Rubio-Zuazo, V. Collado-Negro, C. Heyman, P. Ferrer, I. da Silva, J. A. Gallastegui, A. Gutiérrez-León, and G. R. Castro, J. Phys.: Conf. Ser. 425, 052005 (2013).

[24] M. Bosman, M. Watanabe, D. T. L. Alexander, and V. J. Keast, Ultramicroscopy 106, 1024 (2006).

[25] See Supplemental Material at http://link.aps.org/supplemental/ 10.1103/PhysRevMaterials.3.104407 for AFM characterization of the films.

[26] J. Gazquez et al., Nano Lett. 11, 973 (2011); R. F. Klie, Y. Ito, S. Stemmer, and N. D. Browning, Ultramicroscopy 86, 289 (2001); Y. Ito, R. F. Klie, N. D. Browning, and T. J. Mazanec, J. Am. Ceram. Soc. 85, 969 (2002).

[27] J. Gazquez, S. Bose, M. Sharma, M. A. Torija, S. J. Pennycook, C. Leighton, and M. Varela, APL Mater. 1, 012105 (2013).

[28] Y. M. Kim, J. He, M. D. Biegalski, H. Ambaye, V. Lauter, H.M. Christen, S. T. Pantelides, S. J. Pennycook, S. V. Kalinin, and A. Y. Borisevich, Nat. Mater. 11, 888 (2012); D. O. Klenov, W. Donner, B. Foran, and S. Stemmer, Appl. Phys. Lett. 82, 3427 (2003); S. Stemmer, A. J. Jacobson, X. Chen, and A. Ignatiev, J. Appl. Phys. 90, 3319 (2001); A. Y. Borisevich, A. N. Morozovska, Y. M. Kim, D. Leonard, M. P. Oxley, M. D. Biegalski, E. A. Eliseev, and S. V. Kalinin, Phys. Rev. Lett. 109, 065702 (2012).

[29] H. Fujishiro, Y. Noda, K. Akuzawa, T. Naito, A. Ito, T. Goto, M. Marysko, Z. Jirak, J. Hejtmanek, and K. Nitta, J. Appl. Phys. 121, 115104 (2017).

[30] Y. Noda, H. Fujishiro, T. Naito, A. Ito, T. Goto, J. Hejtmanek, and Z. Jirak, AIP Adv. 6, 025318 (2016).

[31] J. Fujioka, Y. Yamasaki, H. Nakao, R. Kumai, Y. Murakami, M. Nakamura, M. Kawasaki, and Y. Tokura, Phys. Rev. Lett. 111, 027206 (2013).

[32] J. Walter, S. Bose, M. Cabero, G. Yu, M. Greven, M. Varela, and C. Leighton, Phys. Rev. Mater. 2, 111404(R) (2018).

[33] J. Padilla-Pantoja, J. Herrero-Martín, X. Torrelles, B. Bozzo, J. Blasco, C. Ritter, and J. L. García-Muñoz, J. Appl. Phys. 115, 17D721 (2014).

[34] A. Gulec, D. Phelan, C. Leighton, and R. F. Klie, ACS Nano 10, 938 (2016). 